\title{
STRATEGY TO OPTIMIZE THE INFILL STRUCTURE OF THE PART PRODUCED BY FUSED DEPOSITION MODELLING PROCESS
}

\author{
Sashi Kiran MADUGULA ${ }^{1}$, Laurence GIRAUD-MOREAU ${ }^{2}$, Pierre-Antoine \\ ADRAGNA ${ }^{3}$ \\ ${ }^{123}$ ICD-LASMIS, \\ Université de Technologie de Troyes \\ 12 Rue Marie Curie, CS 42060,10004 Troyes Cedex, France \\ ${ }^{1}$ sashi-kiran.madugula@utt.fr, \\ 2laurence.moreau@utt.fr, \\ 3pierre antoine.adragna@utt.fr
}

Key words: Infill structure, 3D printing, Additive manufacturing, Remeshing, Finite element simulation (FE simulation).

\begin{abstract}
The objective of this paper is to optimise the internal structure of 3D printed parts produced by Fused Deposition Modelling (FDM) process. In 3D printing, the term infill refers to the internal structure of the part. The infill design is generally uniform throughout the part. In this paper, we propose a methodology based on an iterative process using remeshing techniques coupled to Finite Element simulation (FE simulation) to control the internal structure of the part without changing the contour. The aim of this methodology is to reinforce the part in the area where the mechanical strength must be improved in order to strengthen the structure, but also to minimize the amount of material in order to minimize the printing time. The proposed method is integrated in a computational environment using the ABAQUS (6.14) and the BL2D adaptive mesher. To validate this methodology, it has been applied on a structure subjected to four point bending. The material used for this structure is PLA (Poly lactic acid).
\end{abstract}

\section{INTRODUCTION}

Additive Manufacturing is a manufacturing process that began to emerge in the 1980s with stereolithography and makes possible to additively manufacture a part layer by layer. Today, this method is a very interesting alternative to conventional manufacturing processes and is particularly well suited in the case of single parts, small series or complex geometry shapes such as lattice structures [2]. Among the various additive manufacturing processes, the most commonly applied [3] and the most affordable is the additive manufacturing by hot wire deposition called FDM (Fused Deposition Modelling). It consists in depositing a thermoplastic material on a support to build a part layer by layer. The material is packaged in the form of a coil of wire or granulate and its composition varies for each polymer (Ex: Poly-Lactic Acid (PLA), Acrylonitrile Butadiene Styrene (ABS), Polyethylene Terephthalate Glycol (PETG), Acrylonitrile Styrene Acrylate (ASA), etc.). 
The FDM process offers the capability to produce parts of complex external shapes but also complex internal geometries (infill) such as cellular or lattice structure. The main benefit of using lattice structure is the decrease of both material weight and production time while keeping a correct strength of the part resulting in cost reductions. Commonly, the slicing software create the infill design with specific volume fractions (see Figure 1). Infill percentage in the part influences the printing time, weight of the part and mechanical stiffness of the part. Optimizing the strength-to-weight ratio is therefore one of the principal research objectives. Several studies have investigated the effect of different infill pattern and infill density on mechanical strength[4],[5]. Cho et al. [6] investigated different infill patterns (zigzag, grid and triangle). Among these three infill patterns, the triangle pattern has given the highest strength. However, these infill designs are uniform throughout the part and therefore not necessarily optimum because not all the zones undergo the same mechanical stress during loading. Hence, localized mechanical stresses are not considered.

Several methods have been proposed to generate different internal structures (infill design) in order to reduce material usage and increase mechanical stiffness. However, it is rare to find works deyoted to both increase of mechanical strength of the part and keeping the printing parameter at the same time. Park et al. [7] and Chen et al. [8] developed lattice cells for the internal structures of part, which allows reducing the material usage. On other hand, Cheng et al [9] and Chen et al. [8] proposed variable cross-sectional area of lattice infill depending on the stress undergone. However, difficulties exists while printing the part using FDM process due to the variable size of the infill.

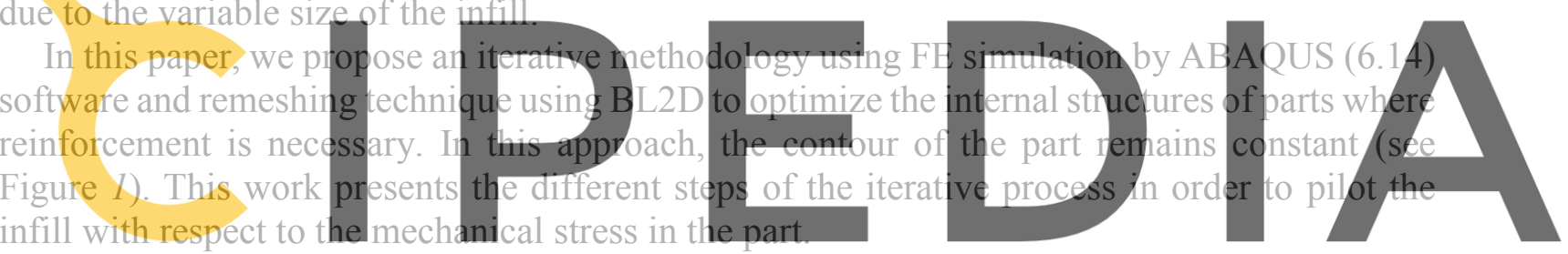

Register for free at https//www.scipedia.com to download the version without the watermark

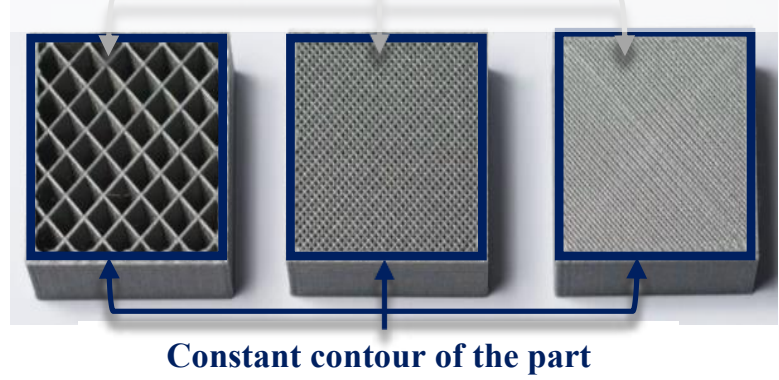

Figure 1: Infill of $20 \%$ (left), $60 \%$ (middle) and $100 \%$ infill (right)

\section{METHODOLOGY}

The key idea of the method proposed in this paper is to use FE simulations and remeshing techniques in order to optimize the infill design of $3 \mathrm{D}$ printed parts. All the internal zones of 
the printed part do not undergo the same mechanical stress during loading. Therefore, it is interesting to use FE results coupled with remeshing technique in order to define a non-uniform infill design keeping the maximum Von-Mises stress of the part below Yield stress $\left(\sigma_{\text {yield Stress }}\right)$ limit of the material (PLA).
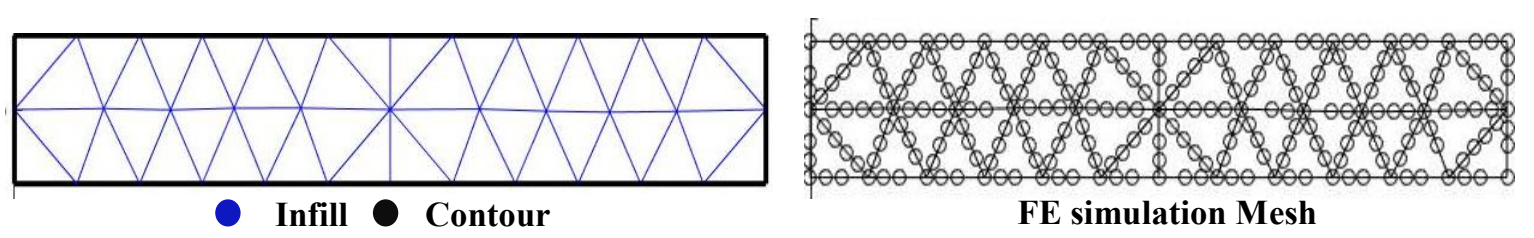

Figure 2: infill and its contour (left) and finite element meshing (right)

It is important to note that the remeshing only concerns with the infill structure of the part. It is purely geometrical and should not be confused with the mesh of the finite element study, generally used to obtain a more precise solution (see Figure 2). Here the remeshing means to create denser infill in areas of high stress and lighter (coarse) infill in the areas of low stress. As a result, the part is strengthened in high stress regions. Remeshing is done using the BL2DV2 remeshing software[1].
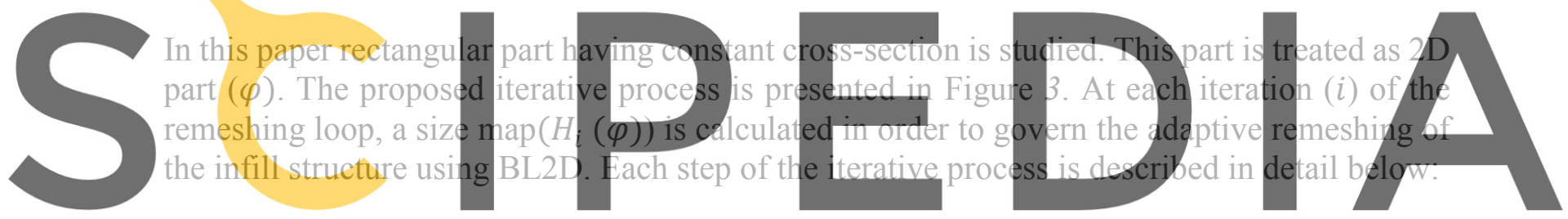

Register for free at https//www.scipedia.Comitudownload the version without the watermark

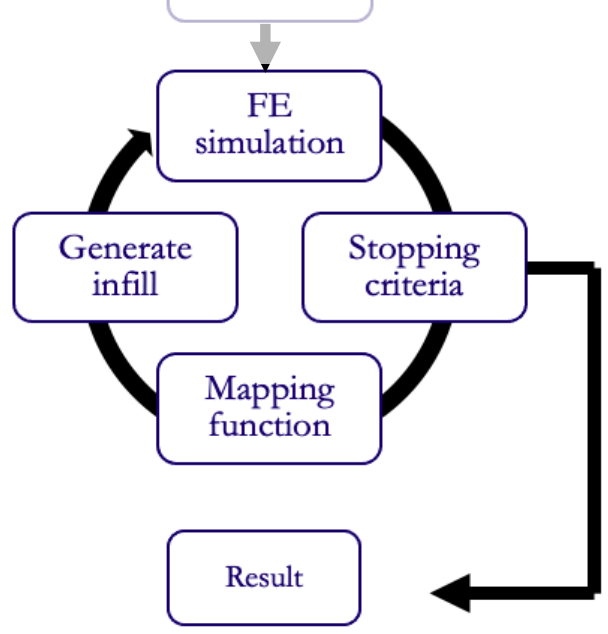

Figure 3: Steps in iterative process 
2.1 Initial infill structure: The first step consists in the generation of the part with initial infill design $\left(M_{i=1}\right)$ using the defined contour. A homogeneous infill is generated as initial infill design. Triangular elements are used for the infill due to their strength compared to other infill geometries [6]. The initial size of the triangular infill is set to a minimal length $\left(L_{\min }\right)=2 \mathrm{~mm}$. The value $2 \mathrm{~mm}$ is based on the $3 \mathrm{D}$ printer nozzle width.

2.2 FE simulation: The proposed method is an iterative procedure, which evolves automatically, without any user intervention. It was then important to develop a MATLAB program to create the input file for ABAQUS (6.14) at each iteration, taking into account the new infill design of the part. The purpose of this step is to determine the mechanical stresses and strains of the part subjected to a given loading conditions, for a given internal geometry. FE simulation is carried out with ABAQUS (6.14) (implicit analysis). 2D beam elements (B21, Timoshenko beam) are used to mesh the part. An elastic model is used for the FE simulation. PLA (Poly Lactic Acid) is used as material and its properties are defined in Table 1.

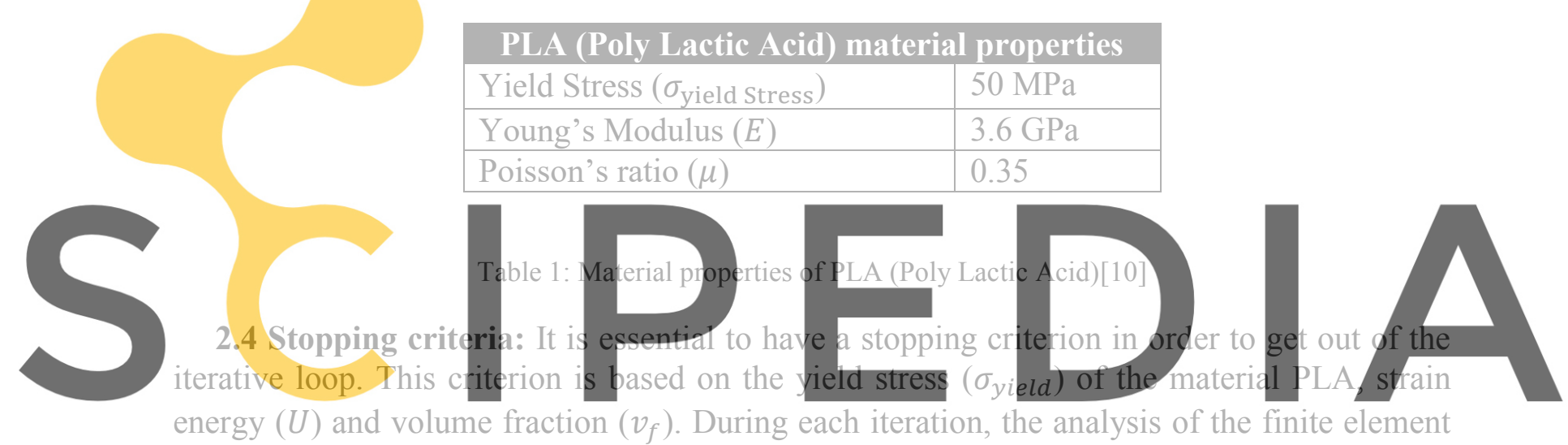

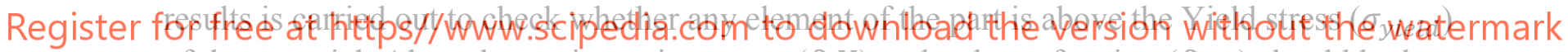
of the material. Also, change in strain energy $(\delta U)$ and volume fraction $\left(\delta v_{f}\right)$ should be less than $5 \%$ (considered as threshold in our case). Once these three criteria are satisfied, then the iterative procedure ends.

2.3 Mapping function: In order to generate the new infill structure (new mesh $M_{i+1}$ ), a size map $\left(H_{i}(\varphi)\right)$ must be defined. The method proposed in this section consists in combination of two size maps. A first size map $\left(H_{i}^{1}(\varphi)\right)$ associated to the existing size of the infill structure $\left(M_{i}\right)$ obtained from BL2D. The size $\left(h_{i}^{1}(p)\right)$ of each node $(p)$ contains average of the size of each element sharing that node $(p)$. Second size map $\left(H_{i}^{2}(\varphi)\right)$ is defined after each FE simulation using ABAQUS (6.14). In order to define size $\left(h_{i}^{2}(p)\right)$ of each node $(p)$, an interpolation is first realized to transfer the stress from the element into the nodes. The vonMises stress value $\left(\sigma_{v m}\right)$ at each node $(p)$ is obtained by calculating the average value of the von-mises stress of all the elements shared by that node $(p)$. For each node $(p)$, if the VonMises stress $\left(\sigma_{v m}\right)$ is less than the yield stress $\left(\sigma_{y i e l d}\right)$, the size of node $\left(h_{i}^{2}(p)\right)$ is calculated using equation 1 . Else, the size of node $\left(h_{i}^{2}(p)\right)$ is calculated using equation 2 . 


$$
\text { Size of node }\left(h_{i}^{2}(p)\right)=L_{\min } *\left(\frac{\sigma_{y i e l d}}{\sigma_{v m}}\right)
$$$$
\text { Size of node }\left(h_{i}^{2}(p)\right)=L_{\text {min }}
$$

equation 1

equation 2

The final size map $\left(H_{i}(\varphi)\right)$ used to control the remeshing is a combination of the two size maps $\left(H_{i}^{1}(\varphi)\right)$ and $\left(H_{i}^{2}(\varphi)\right)$ according to the following formula:

Final size map $\left(H_{i}(\varphi)\right)=5 \%$ of size map $\left(H_{i}^{2}(\varphi)\right)+95 \%$ of size map $\left(H_{i}^{1}(\varphi)\right)$ equation 3

According to equation 3 , only $5 \%$ of the size map $\left(H_{i}^{2}(\varphi)\right)$ is transferred for the next iteration, whereas remaining $95 \%$ is conserved from size $\operatorname{map}\left(H_{i}^{1}(\varphi)\right)$. This will lead to a smoother evolution of the infill design. The proportions of 5\% of size map $\left(H_{i}^{2}(\varphi)\right)$ and $95 \%$ of size $\operatorname{map}\left(H_{i}^{1}(\varphi)\right)$ are chosen arbitrarily to ensure fast convergence and avoid severe size oscillation.

2.4 Generate new infill: Conforming to the size map $\left(H_{i}(\varphi)\right)$, BL2D generates new infill structure $\left(M_{i+1}\right)$ of the part keeping the contour of the part constant. Thereafter, ".inp" file is created for the part with evolved infill structure for the FE Simulation and the loop continues until the stopping criteria is satisfied.
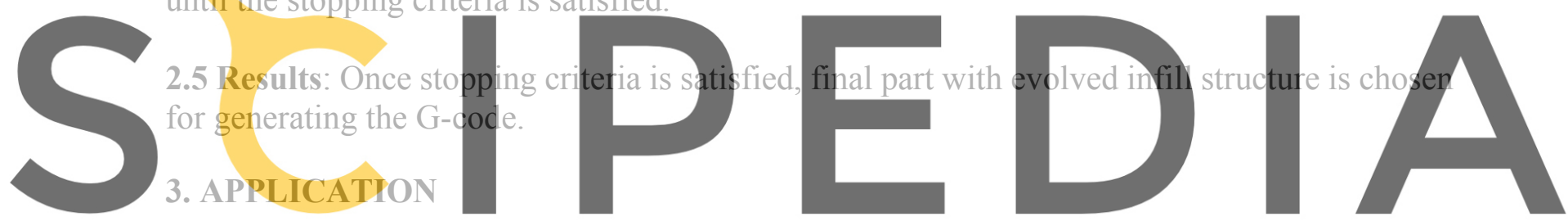

To demonstrate the effectiveness of the proposed iterative method, a rectangular structure

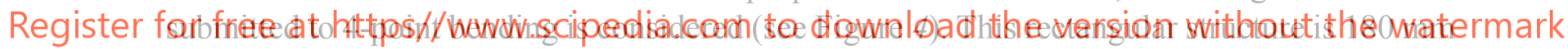

in length, $30 \mathrm{~mm}$ in height and $20 \mathrm{~mm}$ in depth. Two concentrated loads of 500N each are

applied on the top of the structure, symmetrically separated by $90 \mathrm{~mm}$ distance. As boundary conditions roller and a fixed joint applied in the bottom right, and bottom left side of the structure respectively.

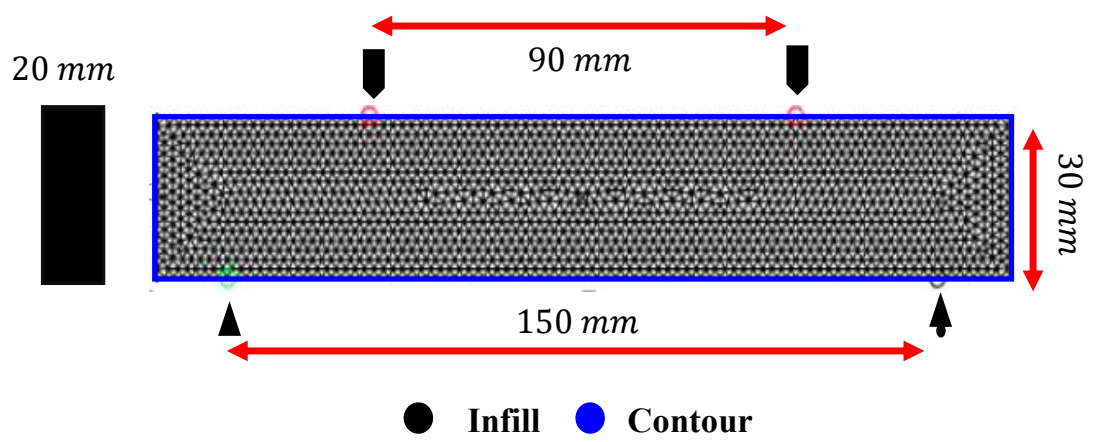

Figure 4: Initial infill design of the part with cross-sectional view 
2.4 Results: For this structure, five iterations were necessary to reach to the final structure and the infill design at each iteration are presented in the Table 3. Table 2 presents the numerical results at each iteration.

\begin{tabular}{|c|c|c|c|c|}
\hline Iteration & $\begin{array}{c}\text { Maximum Von- } \\
\text { Mises stress in } \\
\text { the part }\left(\sigma_{v m}\right) \\
\text { MPa }\end{array}$ & $\begin{array}{l}\text { Maximum } \\
\text { Displacement } \\
(\delta) \mathrm{mm}\end{array}$ & $\begin{array}{c}\text { Elastic strain } \\
\text { energy }(u) \\
\mathbf{m J}\end{array}$ & $\begin{array}{l}\text { Volume } \\
\text { fraction } \\
\left(v_{f}\right)\end{array}$ \\
\hline 1 & 36.022 & 1.00 & 361.12 & 0.70 \\
\hline 2 & 36.533 & 1.18 & 401.56 & 0.55 \\
\hline 3 & 39.738 & 1.26 & 431.81 & 0.48 \\
\hline 4 & 38.8 & 1.33 & 455.68 & 0.45 \\
\hline 5 & 38.3 & 1.37 & 469.54 & 0.42 \\
\hline
\end{tabular}

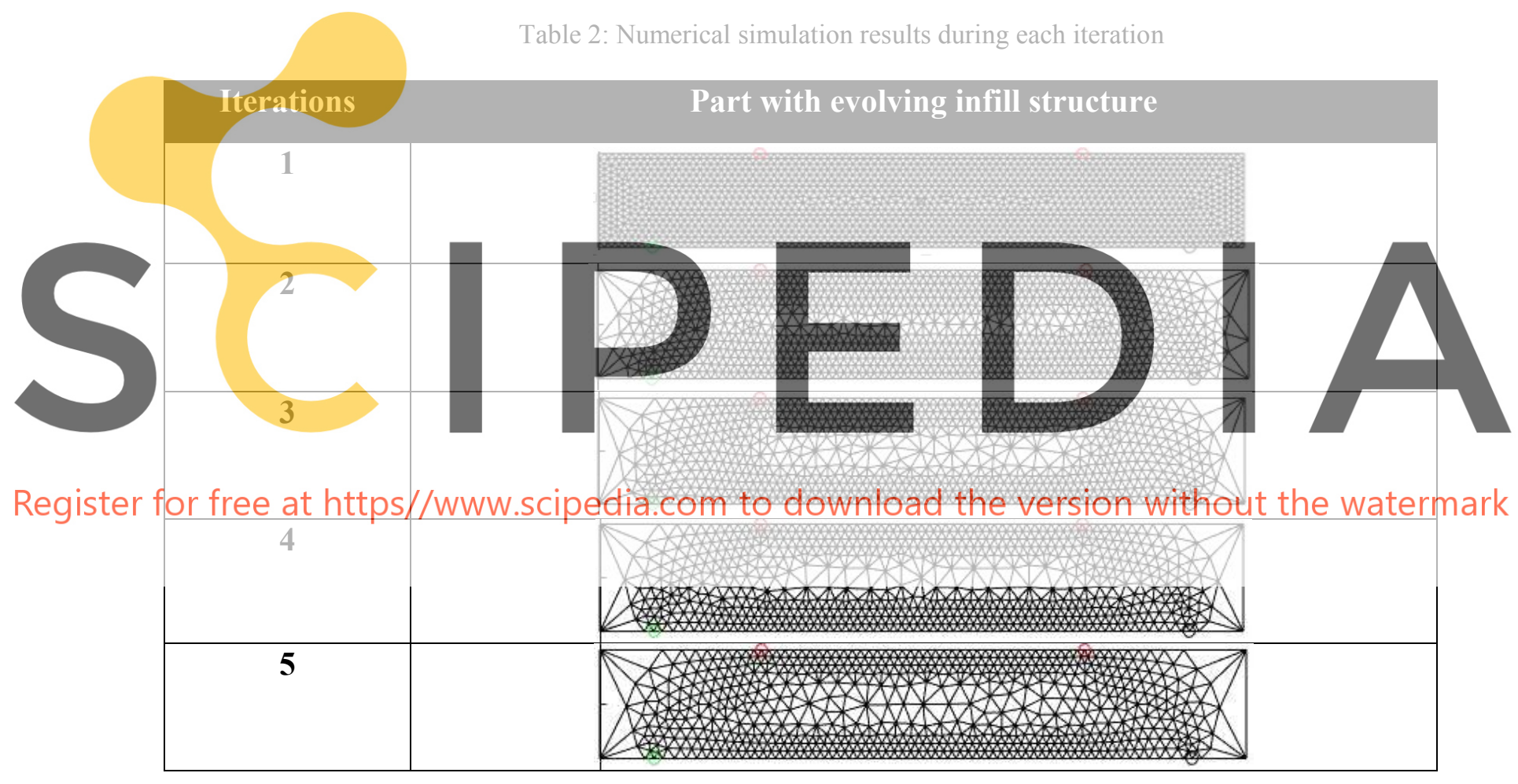

Table 3: Evolution of infill during each iteration

The final infill design generated after five iterations is given in Figure 5. This final structure satisfies the stopping criteria keeping maximum stress of the part within the yield stress ( $\sigma_{\text {yield stress }}$ ) of the material (see Table 1 ). There is also $28 \%$ decrease in volume fraction $\left(v_{f}\right)$ compared to the part of the iteration 1. This final structure obtained (Figure 5) can be used to create the G-code for the 3D-printer. 


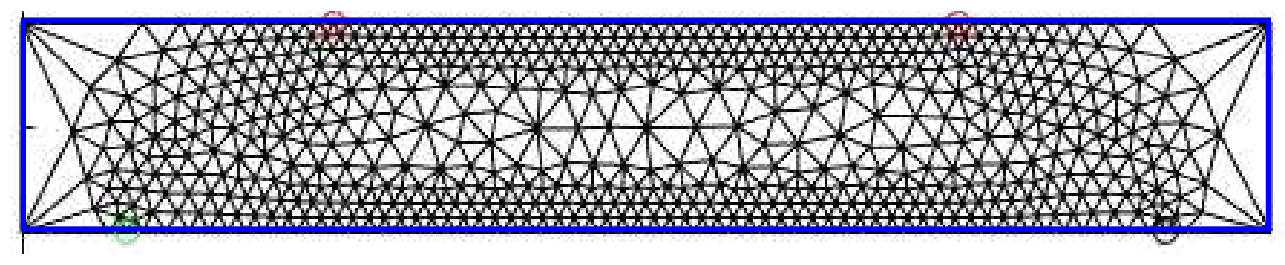

Infill Contour

Figure 5: Final part with infill design after five iterations.

\section{CONCLUSIONS}

This paper gives a new methodology to define infill design of FDM 3D printed parts. It is based on an iterative technique using FE simulation and remeshing technique. A structure submitted to four point bending conditions is used to illustrate this methodology. It is shown that this proposed method makes possible to keep the maximum Von-Mises stress $\left(\sigma_{v m}\right)$ of the part below the Yield stress ( $\sigma_{\text {yield stress }}$ ) of the material (PLA) by using less infill and hence will reduce the printing time.

\section{REFERENCES}

[1] P. Laug and H. Borouchaki, "The BL2D Mesh Generator : Beginner's Guide, User's and
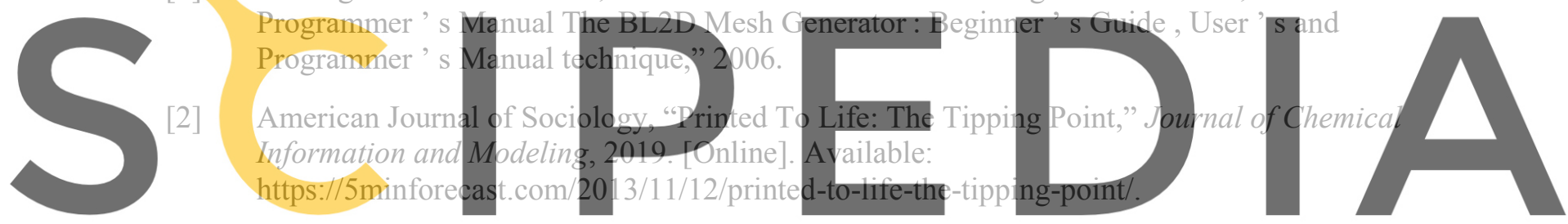

[3] Palermo E (2013) Fused deposition modeling: most common 3d printing method., "No Title."

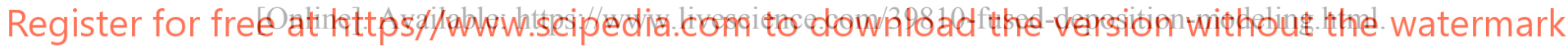

[4] J. T. Cantrell et al., "Experimental characterization of the mechanical properties of 3D-printed ABS and polycarbonate parts," Rapia Prototyp. J., vol. 23, no. 4, pp. 811-824, 2017.

[5] S. Rohde et al., "Experimental Characterization of the Shear Properties of 3D-Printed ABS and Polycarbonate Parts," Exp. Mech., vol. 58, no. 6, pp. 871-884, 2018.

[6] E. Cho and T. Tran, "Investigation on Influence of Infill Pattern and Layer Thickness on Mechanical Strength of PLA Material in 3D Printing Technology Backstepping Control-Based Learn-to-Fly Algorithm for Aircraft Roll Dynamics View project Development of an automatic system," vol. 3, no. September, pp. 27-37, 2019.

[7] S. I. Park, D. W. Rosen, S. kyum Choi, and C. E. Duty, "Effective mechanical properties of lattice material fabricated by material extrusion additive manufacturing," Addit. Manuf., vol. 1, pp. 12-23, 2014.

[8] W. Chen, X. Zheng, and S. Liu, "Finite-Element-Mesh Based Method for Modeling and Optimization of Lattice Structures for Additive Manufacturing," Materials (Basel)., vol. 11, no. 11, p. 2073, 2018.

[9] L. Cheng, J. Bai, and A. C. To, "Functionally graded lattice structure topology optimization for 
the design of additive manufactured components with stress constraints," Comput. Methods Appl. Mech. Eng., vol. 344, pp. 334-359, 2019.

[10] B. Wittbrodt and J. M. Pearce, "The effects of PLA color on material properties of 3-D printed components," Addit. Manuf., vol. 8, pp. 110-116, 2015.
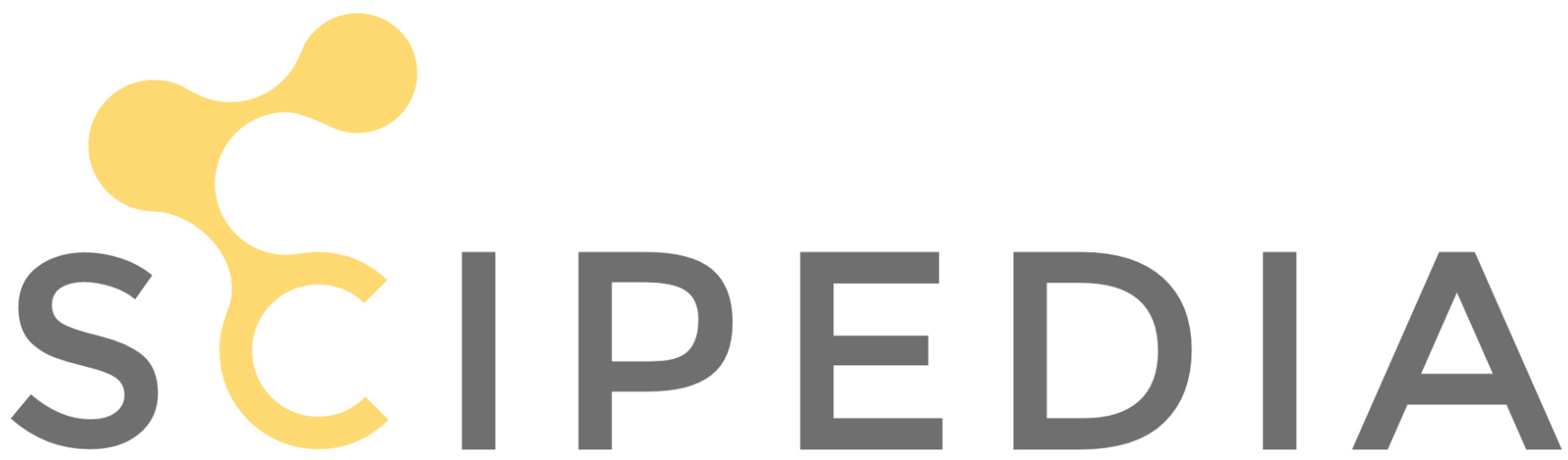

Register for free at https//www.scipedia.com to download the version without the watermark 